
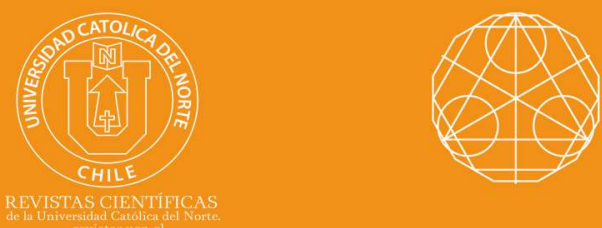

\title{
Oscillation results for a certain class of fourth- order nonlinear delay differential equations
}

\section{Osama Moaaz ${ }^{1}$ @ orcid.org/0000-0003-3850-1022 \\ Clemente Cesarano²® orcid.org/0000-0002-1694-7907}

${ }^{1}$ Mansoura University, Dept. of Mathematics, Mansoura, Egypt.

口o_moaaz@mans.edu.eg

${ }^{2}$ International Telematic University "Uninettuno", Section of Mathematics, Roma, Italy.

¿.cesarano@uninettunouniversity.net

\section{Received: February 2020 | Accepted: September 2020}

\section{Abstract}

In this work, we study the oscillation of the fourth order neutral differential equations with delay argument. By means of generalized Riccati transformation technique, we obtain new oscillation criteria for oscillation of this equation. An example is given to clarify the main results in this paper.

Keywords: Oscillation; Fourth order; Neutral delay; Differential equations.

MSC (2020): 34K10, 34K11.

\section{Cite this article as (IEEE citation style):}

O. Moaaz and C. Cesarano, "Oscillation results for a certain class of fourth-order nonlinear delay differential equations", Proyecciones (Antofagasta, On line), vol. 40, no. 2, pp. 505523, 2021, doi: 10.22199/issn.0717-6279-2021-02-0029

Article copyright: (C) 2021 Osama Moaaz and Clemente Cesarano. This is an open access article distributed under the terms of the Creative Commons License, which permits unrestricted use and distribution provided the original author and source are credited. 


\section{Introduction}

In this paper, we are concerned with the oscillation of the fourth-order nonlinear neutral differential equations with delay argument

$(1.1)\left[r(t)\left((x(t)+p(t) x(\tau(t)))^{\prime \prime \prime}\right)^{\alpha}\right]^{\prime}+q(t) f(x(g(t)))=0, \quad t \geq t_{0}$,

where $r, p, q, \tau, g \in C\left(\left[t_{0}, \infty\right), \mathbf{R}\right), r(t)$ and $q(t)$ are positive, $0 \leq p(t) \leq$ $p<1, \alpha \geq 1$ is a quotient of odd positive integers, $r^{\prime}(t)>0, \tau^{\prime}(t)>0$, $\tau(t) \leq t, g(t) \leq t, \lim _{t \rightarrow \infty} \tau(t)=\infty, \lim _{t \rightarrow \infty} g(t)=\infty$, the function $f$ satisfies the following condition

$$
f \in C(\mathbf{R}, \mathbf{R}), f(x) / x^{\alpha} \geq k>0 \text { for } x=0,
$$

where $k$ a constant and

$$
\int_{t_{0}}^{\infty} r^{-1 / \alpha}(t) d t<\infty
$$

We define the corresponding function $v(t)=x(t)+p(t) x(\tau(t))$. By a solution of Eq. (1.1), we mean a non-trivial real function $x(t) \in C\left(\left[t_{x}, \infty\right)\right)$, $t_{x} \geq t_{0}$, which has the properties $x(t), v(t), v^{\prime}(t), v^{\prime \prime}(t)$ and $r(t)\left[v^{\prime \prime \prime}(t)\right]^{\alpha}$ are continuously differentiable for all $t \in\left[t_{x}, \infty\right)$ and satisfies (1.1) on $\left[t_{x}, \infty\right)$. We consider only those solutions $x(t)$ of $(1.1)$ which satisfy $\sup \{|x(t)|$ : $t \geq L\}>0$ for any $L \geq t_{x}$. A solution of Eq. (1.2) is called oscillatory if it has arbitrary large zeros, otherwise it is called nonoscillatory.

In models concerning chemical, biological and physical phenomena, fourth-order differential equations naturally appear; see [1]. In the past decade, there has been increasing interest in obtaining oscillation and nonoscillation criteria of different classes of third and fourth-order differential equations. The works $[2,3,4,5,6,7,8,9,10,11,12,13]$ improved the oscillation criteria for second-order equations with delay and advanced argument. For even-order delay equations, papers $[15,16,17,18,19,20,21,22,23,24,25,26$, 27] developed the oscillation criteria. Whereas, the results in $[31,32,33,34$, $35,36]$ dealt with the issue of oscillation of equations of odd-order.

Agarwal et al. [14] studied the oscillatory behavior of the delay equation

$$
\left[r_{3}(t)\left(\left[r_{2}(t)\left(\left[r_{1}(t)\left(x^{\prime}(t)\right)^{\alpha_{1}}\right]^{\prime}\right)^{\alpha_{2}}\right]^{\prime}\right)^{\alpha_{3}}\right]^{\prime} \pm q(t) f(x(g(t)))=0,
$$

where $\int^{\infty} r_{i}^{1 / \alpha_{i}}(\vartheta) d \vartheta<\infty, i=1,2,3$. Grace et al. [16] established new criteria for oscillation of delay equation

$$
\left(r(t)\left(x^{\prime}(t)\right)^{\alpha}\right)^{\prime \prime \prime}+q(t) f(x(g(t)))=0,
$$


$\mathrm{Wu}[30]$ and Kamo and Usami [24] studied the oscillation behavior of delay equation

$$
\left(r(t)\left|x^{\prime \prime}(t)\right|^{\alpha-1} x^{\prime \prime}(t)\right)^{\prime \prime}+q|x(t)|^{\beta-1} x(t)=0,
$$

where $\alpha, \beta>0$.

For neutral delay equations, Li et al. [19] studied oscillatory properties of solutions of even-order equations

$$
(x(t)+p(t) x(\tau(t)))^{(n)}+q(t) x(g(t))=0,
$$

relating oscillation of higher-order equations to that of a pair of associated first-order delay differential equations. Based on the comparison with firstorder delay equations, Moaaz et al. [28] established an oscillation criterion for neutral equations

$$
\left(r(t)\left((x(t)+p(t) x(\tau(t)))^{(n-1)}\right)^{\alpha}\right)^{\prime}+q(t) x^{\alpha}(g(t))=0,
$$

where $\int^{\infty} r^{1 / \alpha}(\vartheta) d \vartheta=\infty$..

Actually, we have greatly less results for fourth order neutral differential equations than those can be found in the literature on the oscillation of solutions of differential equations of first, second orders, also even-order delay equations. So, the main objective of this paper is to shed light on the class of fourth order neutral equations (1.1), and through study the oscillation criteria of solutions of this equation. In order to discuss our main results, we need the following lemmas

Lemma 1.1. [3, Lemma 2.1] Assume that $\alpha \geq 1$ is a quotient of odd positive integers, $A, B, U$ and $V$ are constants, $A B \geq 0$ and $V>0$. Then

$$
A^{(\alpha+1) / \alpha}-(A-B)^{(\alpha+1) / \alpha} \leq \frac{1}{\alpha} B^{1 / \alpha}((\alpha+1) A-B)
$$

and

$$
U y-V y^{(\alpha+1) / \alpha} \leq \frac{\alpha^{\alpha}}{(\alpha+1)^{\alpha+1}} U^{\alpha+1} V^{-\alpha}, \quad y \geq 0 .
$$

Lemma 1.2. [37] If the function $x$ satisfies $x^{(i)}(t)>0, i=0,1, \ldots, n$, and $x^{(n+1)}(t)<0$, then 


$$
\frac{x(t)}{t^{n} / n !} \geq \frac{\lambda x^{\prime}(t)}{t^{n-1} /(n-1) !}
$$

for all $\lambda=(0,1)$.

Lemma 1.3. [2, Lemma 2.2.3] Let $x \in C^{n}\left(\left[t_{0}, \infty\right),(0, \infty)\right)$. Assume that $x^{(n)}(t)$ is of fixed sign and not identically zero on $\left[t_{0}, \infty\right)$ and that there exists a $t_{1} \geq t_{0}$ such that

$x^{(n-1)}(t) x^{(n)}(t) \leq 0$ for all $t \geq t_{1}$. If $\lim _{t \rightarrow \infty} x(t) \neq 0$, then for every $\mu \in(0,1)$ there exists $t_{\mu} \geq t_{1}$ such that

$$
\mathrm{x}(t) \geq \frac{\mu}{(n-1) !} t^{n-1}\left|x^{(n-1)}(t)\right| \text { for } t \geq t_{\mu} .
$$

\section{Main results}

In this section, we will establish some oscillation criteria for solutions of the Eq. (1.1). For simplicity, denote by $S^{\oplus}$ the set of all eventually positive solutions of the equation (1.1). Also, we introduce the following notations:

$$
\eta_{0}(t)=\int_{t}^{\infty} r^{-1 / \alpha}(s) d s, \eta_{i}(t)=\int_{t}^{\infty} \eta_{i-1}(s) d s, \eta_{0}(t)=\eta(t), i=1,2,
$$
and

$$
\beta=\frac{\alpha^{\alpha}}{(\alpha+1)^{\alpha+1}}
$$

Lemma 2.1. If $x(t)$ is an eventually positive three times continuously differentiable function such that $r(t)\left(v^{\prime \prime \prime}(t)\right)^{\alpha}$ is continuously differentiable and $\left(r(t)\left(v^{\prime \prime \prime}(t)\right)^{\alpha}\right)^{\prime} \leq 0$ for large $t$, then one of the following cases holds for large $t$,

$$
\begin{array}{ll}
\left(\mathbf{C}_{1}\right) & v(t)>0, v^{\prime}(t)>0, v^{\prime \prime}(t)>0 \text { and } v^{\prime \prime \prime}(t)>0, \\
\left(\mathbf{C}_{2}\right) & v(t)>0, v^{\prime}(t)>0, v^{\prime \prime}(t)<0 \text { and } v^{\prime \prime \prime}(t)>0, \\
\left(\mathbf{C}_{3}\right) & v(t)>0, v^{\prime}(t)<0, v^{\prime \prime}(t)>0 \text { and } v^{\prime \prime \prime}(t)<0, \\
\left(\mathbf{C}_{4}\right) & v(t)>0, v^{\prime}(t)>0, v^{\prime \prime}(t)>0 \text { and } v^{\prime \prime \prime}(t)<0 .
\end{array}
$$

The proof is immediate and hence is omitted.

Lemma 2.2. Let $x(t) \in S^{\oplus}$ and the corresponding $v(t)$ satisfy $\left(\mathbf{C}_{1}\right)$. Then there exist a positive function $\rho_{1} \in C^{1}\left(\left[t_{0}, \infty\right)\right)$ such that for some $\mu \in(0,1)$, 


$$
R_{1}:=\int_{t_{0}}^{\infty}\left(\Psi(s)-\frac{2^{\alpha}}{(\alpha+1)^{\alpha+1}} \frac{r(s)\left(\rho_{1}^{\prime}(s)\right)^{\alpha+1}}{\mu^{\alpha} s^{2 \alpha} \rho_{1}^{\alpha}(s)}\right) d s<\infty
$$

where

$$
\Psi(t)=k \rho_{1}(t) q(t)(1-p(g(t)))^{\alpha}\left(\frac{g(t)}{t}\right)^{3 \alpha}
$$

Proof. Assume that $x(t) \in S^{\oplus}$. It follow from the facts $r^{\prime}(t)>0$ and $\left(r(t)\left(v^{\prime \prime \prime}(t)\right)^{\alpha}\right)^{\prime} \leq 0$ that $v^{(4)}(t)<0$. Since $\tau(t) \leq t$ and $v^{\prime}(t)>0$, we get

$$
\begin{aligned}
x(t) & =v(t)-p(t) x(\tau(t)) \\
& \geq(1-p(t)) v(t) .
\end{aligned}
$$

From equation (1.1), we see that

$$
\begin{aligned}
\left(r(t)\left(v^{\prime \prime \prime}(t)\right)^{\alpha}\right)^{\prime} & =-q(t) f(x(g(t))) \\
& \leq-k q(t)(1-p(g(t)))^{\alpha} v^{\alpha}(g(t)) .
\end{aligned}
$$

Now, we define a generalized Riccati substitution by $\omega(t):=\rho_{1}(t) \frac{r(t)\left(v^{\prime \prime \prime}(t)\right)^{\alpha}}{v^{\alpha}(t)}$.

Then $\omega(t)>0$. By differentiating and using (2.4), we obtain

$$
\begin{aligned}
\omega^{\prime}(t) \leq & \frac{\rho_{1}^{\prime}(t)}{\rho_{1}(t)} \omega(t)-k \rho_{1}(t) q(t)(1-p(g(t)))^{\alpha} \frac{v^{\alpha}(g(t))}{v^{\alpha}(t)} \\
& -\alpha \rho_{1}(t) \frac{r(t)\left(v^{\prime \prime \prime}(t)\right)^{\alpha}}{v^{\alpha+1}(t)} v^{\prime}(t) .
\end{aligned}
$$

From Lemma 1.2, we have that $v(t) \geq \frac{t}{3} v^{\prime}(t)$, and hence,

$$
\frac{v(g(t))}{v(t)} \geq \frac{g^{3}(t)}{t^{3}}
$$

It follows from Lemma 1.3 that

$$
v^{\prime}(t) \geq \frac{\mu}{2} t^{2} v^{\prime \prime \prime}(t)
$$

for all $\mu \in(0,1)$ and every sufficiently large $t$. Thus, by (2.6), (2.7) and (2.8), we get 


$$
\begin{aligned}
\omega^{\prime}(t) \leq & \frac{\rho_{1}^{\prime}(t)}{\rho_{1}(t)} \omega(t)-k \rho_{1}(t) q(t)(1-p(g(t)))^{\alpha}\left(\frac{g(t)}{t}\right)^{3 \alpha} \\
& -\alpha \mu \frac{t^{2}}{2 r^{1 / \alpha}(t) \rho_{1}^{1 / \alpha}(t)} \omega^{\frac{\alpha+1}{\alpha}}(t) .
\end{aligned}
$$

Using the inequality (1.5) with $U=\frac{\rho_{1}^{\prime}(t)}{\rho_{1}(t)}, V=\frac{\alpha \mu t^{2}}{2 r^{1 / \alpha}(t) \rho_{1}^{1 / \alpha}(t)}$ and $y=\omega$, we get

$$
\omega^{\prime}(t) \leq-\Psi(t)+\frac{2^{\alpha}}{(\alpha+1)^{\alpha+1}} \frac{r(t)\left(\rho_{1}^{\prime}(t)\right)^{\alpha+1}}{\mu^{\alpha} t^{2 \alpha} \rho_{1}^{\alpha}(t)} .
$$

This implies that

$$
\int_{t_{1}}^{t}\left(\Psi(s)-\frac{2^{\alpha}}{(\alpha+1)^{\alpha+1}} \frac{r(s)\left(\rho_{1}^{\prime}(s)\right)^{\alpha+1}}{\mu^{\alpha} s^{2 \alpha} \rho_{1}^{\alpha}(s)}\right) d s \leq \omega\left(t_{1}\right) .
$$

This completes the proof of Lemma 2.2.

Lemma 2.3. Let $x(t) \in S^{\oplus}$ and the corresponding $v(t)$ satisfy $\left(\mathbf{C}_{2}\right)$. Then there exist a positive function $\rho_{2} \in C^{1}\left(\left[t_{0}, \infty\right)\right)$ such that

$$
R_{2}:=\int_{t_{0}}^{\infty}\left(\Phi(s)-\frac{\left(\rho_{2}^{\prime}(s)\right)^{2}}{4 \rho_{2}(s)}\right) d s<\infty,
$$

where

$$
\Phi(t)=\rho_{2}(t) \int_{t}^{\infty}\left(\frac{k}{r(u)} \int_{u}^{\infty} q(s) \frac{g^{\alpha}(s)}{s^{\alpha}}(1-p(g(s)))^{\alpha} d s\right)^{1 / \alpha} d u .
$$

Proof. In view of the proof of Lemma 2.2, it follows that (2.4) holds. Integrating (2.4) from $t$ to $u$, we obtain

$$
r(u)\left(v^{\prime \prime \prime}(u)\right)^{\alpha}-r(t)\left(v^{\prime \prime \prime}(t)\right)^{\alpha} \leq-k \int_{t}^{u} q(s)(1-p(g(s)))^{\alpha} v^{\alpha}(g(s)) d s .
$$


From Lemma 1.2, we get that $v(t) \geq t v^{\prime}(t)$, and hence,

$$
v(g(t)) \geq \frac{g(t)}{t} v(t) .
$$

For (2.13), letting $u \rightarrow \infty$ and using (2.14), we get

$\mathrm{r}(t)\left(v^{\prime \prime \prime}(t)\right)^{\alpha} \geq k v^{\alpha}(t) \int_{t}^{\infty} q(s) \frac{g^{\alpha}(s)}{s^{\alpha}}(1-p(g(s)))^{\alpha} d s$.

Integrating the former inequality again from $t$ to $\infty$, we get

$$
v^{\prime \prime}(t) \leq-v(t) \int_{t}^{\infty}\left(\frac{k}{r(u)} \int_{u}^{\infty} q(s) \frac{g^{\alpha}(s)}{s^{\alpha}}(1-p(g(s)))^{\alpha} d s\right)^{1 / \alpha} d u .
$$

Now, we define

$$
w(t)=\theta(t) \frac{v^{\prime}(t)}{v(t)} .
$$

Then $w(t)>0$ for $t \geq t_{1} \geq t_{0}$. By differentiating the last inequality and using (2.15), we find

$$
\begin{gathered}
w^{\prime}(t)=\frac{\theta^{\prime}(t)}{\theta(t)} w(t)+\theta(t) \frac{v^{\prime \prime}(t)}{v(t)}-\theta(t)\left(\frac{v^{\prime}(t)}{v(t)}\right)^{2} \\
\leq \frac{\theta^{\prime}(t)}{\theta(t)} w(t)-\theta(t) \int_{t}^{\infty}\left(\frac{k}{r(u)} \int_{u}^{\infty} q(s) \frac{g^{\alpha}(s)}{s^{\alpha}}(1-p(g(s)))^{\alpha} d s\right)^{1 / \alpha} d u \\
-\frac{1}{\theta(t)} w^{2}(t) .
\end{gathered}
$$

Thus, we obtain

$$
w^{\prime}(t) \leq-\Phi(t)+\frac{\left(\theta^{\prime}(t)\right)^{2}}{4 \theta(t)}
$$

Then, we get 


$$
\int_{t_{1}}^{t}\left(\Phi(s)-\frac{\left(\theta^{\prime}(t)\right)^{2}}{4 \theta(t)}\right) d s \leq w\left(t_{1}\right) .
$$

This completes the proof of Lemma 2.3.

Lemma 2.4. Assume that $p(t)=p$ (constant). If $x(t) \in S^{\oplus}$ and the corresponding $v(t)$ satisfy $\left(\mathbf{C}_{3}\right)$, then there exists an odd integer $n \geq 0$ such that

$$
R_{3}:=\int_{t_{0}}^{\infty}\left(k \lambda^{\alpha} \eta_{2}^{\alpha}(s) q(s)-\alpha \beta \frac{\eta_{1}(s)}{\eta_{2}(s)}\right) d s \leq 1,
$$

where $\lambda=\sum_{r=0}^{n}(-1)^{r} p^{r}<1$.

Proof. Let $x(t)$ is a positive solution of equation (1.1). Since $r(t)\left(v^{\prime \prime \prime}(t)\right)^{\alpha}$ nonincreasing, we have that $r(s)\left(v^{\prime \prime \prime}(s)\right)^{\alpha} \leq r(t)\left(v^{\prime \prime \prime}(t)\right)^{\alpha}$ for all $s \geq t \geq$ $t_{1}$. This yields

$$
v^{\prime \prime \prime}(s) \leq\left[r(t)\left(v^{\prime \prime \prime}(t)\right)^{\alpha}\right]^{1 / \alpha} \frac{1}{r^{1 / \alpha}(s)} .
$$

By integrating this inequality three times from $t$ to $\infty$ and using $\left(\mathbf{C}_{3}\right)$, we get

$$
(-1)^{i+1} v^{(i)}(t) \leq\left[r(t)\left(v^{\prime \prime \prime}(t)\right)^{\alpha}\right]^{1 / \alpha} \eta_{2-i}(t), i=0,1,2 .
$$

New, we define

$$
\tau^{0}(t)=t, \tau^{j}(t)=\tau\left(\tau^{j-1}(t)\right), j=1,2, \ldots .
$$

Then, we obtain

$$
\mathrm{x}(t)=v(t)-p x(\tau(t))=v(t)-p v(\tau(t))+p^{2} x\left(\tau^{2}(t)\right),
$$

for all sufficiently large $t$. Repeating this procedure and the monotonicity of $v$, we obtain that there exists an odd integer $n \geq 0$ such that $\tau^{n+1}(g(t)) \geq$ $t_{1} \geq t_{0}$ and

$$
\mathrm{x}(g(t))=\sum_{r=0}^{n}(-1)^{r} p^{r} v\left(\tau^{r}(g(t))\right)+p^{n+1} x\left(\tau^{n+1}(g(t))\right) \geq \lambda v(g(t)),
$$

where $\lambda>0$. Hence, from (1.1), we see that

$$
\begin{aligned}
{\left[r(t)\left(v^{\prime \prime \prime}(t)\right)^{\alpha}\right]^{\prime} } & \leq-k \lambda^{\alpha} q(t) v^{\alpha}(g(t)) \\
& \leq-k \lambda^{\alpha} q(t) v^{\alpha}(t) .
\end{aligned}
$$

Next, we define

$$
\psi(t)=\frac{r(t)\left(v^{\prime \prime \prime}(t)\right)^{\alpha}}{v^{\alpha}(t)}
$$


Thus, we see that $\psi(t)<0$ and satisfies $\psi^{\prime}(t)=\frac{\left[r(t)\left(v^{\prime \prime \prime}(t)\right)^{\alpha}\right]^{\prime}}{v^{\alpha}(t)}-\alpha \frac{r(t)\left(v^{\prime \prime \prime}(t)\right)^{\alpha}}{v^{\alpha+1}(t)} v^{\prime}(t)$.

Hence, from (2.20) and [(2.18) with $i=1]$, we have

$$
\psi^{\prime}(t) \leq-k \lambda^{\alpha} q(t)-\alpha \eta_{1}(t) \psi^{\frac{1+\alpha}{\alpha}}(t) .
$$

From (2.18) with $i=0$, we have

$$
\eta_{2}^{\alpha}(t) \psi(t) \geq-1
$$

Multiplying (2.22) by $\eta_{2}^{\alpha}(t)$ and integrating from $t_{1}$ to $t$, we obtain

$$
\begin{gathered}
\eta_{2}^{\alpha}(t) \psi(t)-\eta_{2}^{\alpha}\left(t_{1}\right) \psi\left(t_{1}\right) \leq-\alpha \int_{t_{1}}^{t} \eta_{1}(s) \eta_{2}^{\alpha-1}(s) \psi(s) d s \\
-k \lambda^{\alpha} \int_{t_{1}}^{t} \eta_{2}^{\alpha}(s) q(s) d s-\alpha \int_{t_{1}}^{t} \eta_{1}(s) \eta_{2}^{\alpha}(s) \psi^{\frac{1+\alpha}{\alpha}}(s) d s
\end{gathered}
$$

which with (2.23) gives

$$
\begin{aligned}
1+\eta_{2}^{\alpha}\left(t_{1}\right) \psi\left(t_{1}\right) \geq & k \lambda^{\alpha} \int_{t_{1}}^{t} \eta_{2}^{\alpha}(s) q(s) d s \\
& +\alpha \int_{t_{1}}^{t} \eta_{1}(s) \eta_{2}^{\alpha-1}(s)\left[\psi(s)+\eta_{2}(s) \psi^{\frac{1+\alpha}{\alpha}}(s)\right] d s .
\end{aligned}
$$

Using the inequality (1.5) with $U=1, V=\eta_{2}(s)$ and $y=-\psi$, we get $\psi(s)+\eta_{2}(s) \psi^{\frac{1+\alpha}{\alpha}}(s) \geq-\frac{\alpha^{\alpha}}{(\alpha+1)^{\alpha+1}} \eta_{2}^{-\alpha}(s)$.

Hence, it follows that

$$
\begin{aligned}
\int_{t_{1}}^{t}\left(k \lambda^{\alpha} \eta_{2}^{\alpha}(s) q(s)-\frac{\alpha^{\alpha+1}}{(\alpha+1)^{\alpha+1}} \frac{\eta_{1}(s)}{\eta_{2}(s)}\right) d s & \leq 1+\eta_{2}^{\alpha}\left(t_{1}\right) \psi\left(t_{1}\right) \\
& \leq 1
\end{aligned}
$$

This completes the proof of Lemma 2.4.

Lemma 2.5. Assume that $x(t) \in S^{\oplus}$ and the corresponding $v(t)$ satisfy $\left(\mathbf{C}_{4}\right)$. Then

$R_{4}:=\int_{t_{0}}^{\infty}\left(\frac{k \widetilde{\mu}^{\alpha}}{2^{\alpha}} q(s)\left[\eta_{0}(s)(1-p(g(s))) g^{2}(s)\right]^{\alpha}-\frac{\alpha \beta}{r^{1 / \alpha}(s) \eta_{0}(s)}\right) d s \leq 1$,

for some $\widetilde{\mu} \in(0,1)$. 
Proof. In view of the proofs of Lemma 2.2 and Lemma 2.4, we have that (2.4) and (2.18) with $i=2$ hold, respectively. The inequality (2.18) with $i=2$ yields

$$
\eta_{0}^{\alpha}(t) \frac{r(t)\left(v^{\prime \prime \prime}(t)\right)^{\alpha}}{\left(v^{\prime \prime}(t)\right)^{\alpha}} \geq-1
$$

From Lemma 1.3, we have that $v(t) \geq \frac{\widetilde{\mu}}{2} t^{2} v^{\prime \prime}(t)$ for all $\widetilde{\mu} \in(0,1)$ and every sufficiently large $t$. Thus, there exists a $t_{2} \geq t_{1}$ such that

$$
\frac{v(g(t))}{v^{\prime \prime}(g(t))} \geq \frac{\widetilde{\mu}}{2} g^{2}(t)
$$

for $t \geq t_{2}$. Next, we define

$$
\varphi(t)=\frac{r(t)\left(v^{\prime \prime \prime}(t)\right)^{\alpha}}{\left(v^{\prime \prime}(t)\right)^{\alpha}} .
$$

We note that $\varphi(t)<0$ for $t \geq t_{1}$. By differentiating (2.27) and using (2.4) and (2.26), we obtain

$$
\varphi^{\prime}(t) \leq-\frac{k \widetilde{\mu}^{\alpha}}{2^{\alpha}} q(t)(1-p(g(t)))^{\alpha} g^{2 \alpha}(t)\left(\frac{v^{\prime \prime}(g(t))}{v^{\prime \prime}(t)}\right)^{\alpha}-\alpha \frac{1}{r^{1 / \alpha}(t)} \varphi^{\frac{\alpha+1}{\alpha}}(t) \text {. }
$$
hence

Since, $g(t) \leq t$ and $v^{\prime \prime \prime}(t)<0$, we have that $v^{\prime \prime}(g(t)) \geq v^{\prime \prime}(t)$, and

$$
\varphi^{\prime}(t) \leq-\frac{k \widetilde{\mu}^{\alpha}}{2^{\alpha}} q(t)(1-p(g(t)))^{\alpha} g^{2 \alpha}(t)-\frac{\alpha}{r^{1 / \alpha}(t)} \varphi^{\frac{\alpha+1}{\alpha}}(t)
$$

Multiplying (2.28) by $\eta_{0}^{\alpha}(t)$ and integrating from $t_{2}$ to $t$, and using (2.25), we obtain

$$
\begin{aligned}
1+\eta_{0}^{\alpha}\left(t_{2}\right) \varphi\left(t_{2}\right) \geq & \frac{k \widetilde{\mu}^{\alpha}}{2^{\alpha}} \int_{t_{2}}^{t} q(s) \eta_{0}^{\alpha}(s)(1-p(g(s)))^{\alpha} g^{2 \alpha}(s) d s \\
& +\alpha \int_{t_{2}}^{t} \frac{\eta_{0}^{\alpha-1}(s)}{r^{1 / \alpha}(s)}\left(\varphi(s)+\eta_{0}(s) \varphi^{\frac{1+\alpha}{\alpha}}(s)\right) d s .
\end{aligned}
$$

By following the same steps in Lemma 2.4, we get that

$$
\begin{aligned}
\int_{t_{2}}^{t}\left(\frac{k \widetilde{\mu}^{\alpha}}{2^{\alpha}} q(s) \eta_{0}^{\alpha}(s)(1-p(g(s)))^{\alpha} g^{2 \alpha}(s)-\left(\frac{\alpha}{\alpha+1}\right)^{\alpha+1}\right. & \left.\frac{1}{r^{1 / \alpha}(s) \eta_{0}(s)}\right) d s \\
& \leq 1+\eta_{0}^{\alpha}\left(t_{2}\right) \varphi\left(t_{2}\right) \\
& \leq 1
\end{aligned}
$$


This completes the proof of Lemma 2.5.

Theorem 2.1. Assume that $p(t)=p$ (constant). If there exist positive functions $\rho, \theta \in C^{1}\left(\left[t_{0}, \infty\right)\right)$ and odd integer $n \geq 0$ such that $R_{1}=R_{2}=\infty$, $R_{3}>1$ and $R_{4}>1$, for some $\mu, \widetilde{\mu} \in(0,1)$, where $R_{1}, R_{2}, R_{3} \$ a n d R_{4}$ are defined by (2.1), (2.11), (2.17) and (2.24), respectively, then every solution of (1.1) is oscillatory.

In the next theorem, we establish new oscillation results for equation (1.1) by using the integral averaging technique due to Philos [38].

Theorem 2.2. Assume that there exist positive functions $\rho, \theta \in C^{1}\left(\left[t_{0}, \infty\right)\right)$, $H_{i}, h_{i} \in C(D, \mathbf{R})$ for $i=1,2,3,4$, where $D=\left\{(t, s) \in \mathbf{R}^{2}: t \geq s \geq t_{0}\right\}$, such that $H_{i}(t, t)=0 \$$ fort $\geq t_{0}, H_{i}(t, s)>0$ for $t>s \geq t_{0}, H_{i}$ has a nonpositive continuous partial derivative $\partial H_{i} / \partial s$ satisfying

$$
\begin{gathered}
\rho(s) \frac{\partial H_{1}(t, s)}{\partial s}+\rho^{\prime}(s) H_{1}(t, s)=h_{1}(t, s) H_{1}^{\frac{\alpha}{\alpha+1}}(t, s), \\
\quad \theta(s) \frac{\partial H_{2}(t, s)}{\partial s}+\theta^{\prime}(s) H_{2}(t, s)=h_{2}(t, s) \sqrt{H_{2}(t, s)}, \\
\rho_{3}(s) \frac{\partial H_{3}(t, s)}{\partial s}+\rho_{3}^{\prime}(s) H_{3}(t, s)=-h_{3}(t, s) H_{3}^{\frac{\alpha}{\alpha+1}}(t, s) \\
\text { and }
\end{gathered}
$$$$
\begin{aligned}
& \rho_{4}(s) \frac{\partial H_{4}(t, s)}{\partial s}+\rho_{4}^{\prime}(s) H_{4}(t, s)=-h_{4}(t, s) H_{4}^{\frac{\alpha}{\alpha+1}}(t, s) . \\
& \text { If }
\end{aligned}
$$

$\limsup _{t \rightarrow \infty} \frac{1}{H_{1}\left(t, t_{0}\right)} \int_{t_{0}}^{t}\left(H_{1}(t, s) \Psi(s)-\frac{2^{\alpha}}{(\alpha+1)^{\alpha+1}} \frac{r(s)\left[h_{1}^{\alpha+1}(t, s)\right]_{+}}{\mu^{\alpha} s^{2 \alpha} \rho^{\alpha}(s)}\right) d s=\infty$,

(2.30) $\limsup _{t \rightarrow \infty} \frac{1}{H_{2}\left(t, t_{0}\right)} \int_{t_{0}}^{t}\left(H_{2}(t, s) \Phi(s)-\frac{\left[h_{2}^{2}(t, s)\right]_{+}}{4 \theta(s)}\right) d s=\infty$

$$
\limsup _{t \rightarrow \infty} \frac{1}{H_{3}\left(t, t_{0}\right)} \int_{t_{0}}^{t}\left(k \lambda^{\alpha} H_{3}(t, s) \rho_{3}(s) q(s)-\frac{1}{(\alpha+1)^{\alpha+1}} \frac{\left[h_{3}^{\alpha+1}(t, s)\right]_{+}}{\eta_{1}^{\alpha} \rho_{3}^{\alpha}(s)}\right) d s=\infty
$$


(2.32) $\limsup _{t \rightarrow \infty} \frac{1}{H_{4}\left(t, t_{0}\right)} \int_{t_{0}}^{t}\left(\Omega(t, s)-\frac{1}{(\alpha+1)^{\alpha+1}} \frac{r h_{4}^{\alpha+1}}{\rho_{4}^{\alpha}}\right) d s=\infty$,

where $\Omega(t, s)=\frac{k \widetilde{\mu}^{\alpha}}{2^{\alpha}} H_{4}(t, s) \rho_{4}(s) q(s)(1-p(g(s)))^{\alpha} g^{2 \alpha}(s)$, then every solution of (1.1) is oscillatory.

Proof. Assume that equation (1.1) has a nonoscillatory solution $x(t)$. Without loss of generality, we may assume that $x(t)$ is an eventually positive solution. By Lemma 2.1, we observe that $v(t)$ has one of the four cases $\left(\mathbf{C}_{1}\right)-\left(\mathbf{C}_{4}\right)$ for $t \geq t_{1}$. For Case $\left(\mathbf{C}_{1}\right)$, proceeding as a proof of Lemma 2.2, we have that $(2.10)$ holds. Multiplying $(2.10)$ by $H_{1}(t, s)$ and integrating the resulting inequality from $t_{1}$ to $t$, we find

$$
\begin{aligned}
\int_{t_{1}}^{t} H_{1}(t,(2) 3 \mathrm{~B})(s) d s \leq & H_{1}\left(t, t_{1}\right) \omega\left(t_{1}\right)+\int_{t_{1}}^{t}\left(\frac{\partial H_{1}(t, s)}{\partial s}+\frac{\rho^{\prime}(s)}{\rho(s)} H_{1}(t, s)\right) \omega(s) d s \\
(2.34) & -\int_{t_{1}}^{t} \frac{\alpha \mu s^{2}}{2 r^{1 / \alpha}(s) \rho^{1 / \alpha}(s)} H_{1}(t, s) \omega^{\frac{\alpha+1}{\alpha}}(s) d s \\
(2.35) \leq & H_{1}\left(t, t_{1}\right) \omega\left(t_{1}\right)+\int_{t_{1}}^{t} \frac{h(t, s)}{\rho(s)} H_{1}^{\frac{\alpha}{\alpha+1}}(t, s) \omega(s) d s \\
(2.36) & -\int_{t_{1}}^{t} \frac{\alpha \mu s^{2}}{2 r^{1 / \alpha}(s) \rho^{1 / \alpha}(s)} H_{1}(t, s) \omega^{\frac{\alpha+1}{\alpha}}(s) d s .
\end{aligned}
$$

By Lemma 1.1, if we set $U=\frac{h}{\rho} H_{1}^{\frac{\alpha}{\alpha+1}}, V=\frac{\alpha \mu s^{2}}{2 r^{1 / \alpha} \rho^{1 / \alpha}} H_{1}$ and $y=\omega$, then we obtain

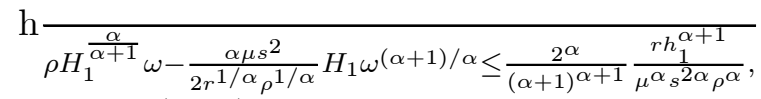

which with (2.36) gives

$$
1 \overline{H_{1}\left(t, t_{1}\right) \int_{t_{1}}^{t}\left(H_{1}(t, s) \Psi(s)-\frac{2^{\alpha}}{(\alpha+1)^{\alpha+1}} \frac{r(s)\left[h_{1}^{\alpha+1}(t, s)\right]_{+}}{\mu^{\alpha} s^{2 \alpha \rho^{\alpha}(s)}}\right) d s \leq \omega\left(t_{1}\right),}
$$

which contradicts (2.29). In the Case $\left(\mathbf{C}_{2}\right)$, as in the proof of Lemma 2.3, we get that (2.16) holds. Multiplying $(2.16)$ by $H_{2}(t, s)$ and integrating the resulting from $t_{2}$ to $t$, we obtain

$$
\int_{t_{2}}^{t} H_{2}(t, s) \Phi(s) d s \leq H_{2}\left(t, t_{2}\right) w\left(t_{2}\right)+\int_{t_{2}}^{t}\left(\frac{\partial H_{2}(t, s)}{\partial s}+\frac{\theta^{\prime}(s)}{\theta(s)} H_{2}(t, s)\right) w(s) d s
$$




$$
\begin{aligned}
& -\int_{t_{2}}^{t} \frac{1}{\theta(s)} H_{2}(t, s) w^{2}(s) d s \\
\leq & H_{2}\left(t, t_{2}\right) w\left(t_{2}\right)+\int_{t_{2}}^{t} \frac{h_{2}(t, s)}{\theta(s)} \sqrt{H_{2}(t, s)} w(s) d s \\
& -\int_{t_{2}}^{t} \frac{1}{\theta(s)} H_{2}(t, s) w^{2}(s) d s
\end{aligned}
$$

and so,

$$
1 \overline{H_{2}\left(t, t_{2}\right) \int_{t_{2}}^{t}\left(H_{2}(t, s) \Phi(s)-\frac{h_{2}^{2}(t, s)}{4 \theta(s)}\right) d s \leq w\left(t_{2}\right),}
$$

which contradicts $(2.30)$. Now, let Case $\left(\mathbf{C}_{3}\right)$ holds. Proceeding as a proof of Lemma 2.4, we have that (2.18), (2.20) and (2.22) hold. Next, we define $\bar{\psi}(t)=\rho_{3}(t) \psi(t)$ where $\psi(t)$ defined as $(2.21)$. Then, we see that $\bar{\psi}(t)<0$ and satisfies $\bar{\psi}^{\prime}(t)=\left(\rho_{3}^{\prime}(t) / \rho_{3}(t)\right) \bar{\psi}(t)+\rho_{3}(t) \psi^{\prime}(t)$. Hence, from (2.22), we find

$$
\bar{\psi}^{\prime}(t) \leq \frac{\rho_{3}^{\prime}(t)}{\rho_{3}(t)} \bar{\psi}(t)-k \lambda^{\alpha} \rho_{3}(t) q(t)-\frac{\alpha \eta_{1}(t)}{\rho_{3}^{1 / \alpha}(t)} \bar{\psi}^{\frac{1+\alpha}{\alpha}}(t) .
$$

Multiplying this inequality by $H_{3}(t, s)$ and integrating the resulting from $t_{3}$ to $t$, we obtain

(2.37) $\int_{t_{3}}^{t} k \lambda^{\alpha} H_{3}(t, s) \rho_{3}(s) q(s) d s$

$$
\begin{gathered}
\leq H_{3}\left(t, t_{3}\right) \bar{\psi}\left(t_{3}\right)+\int_{t_{3}}^{t}\left(\frac{\partial H_{3}(t, s)}{\partial s}+\frac{\rho_{3}^{\prime}(s)}{\rho_{3}(s)} H_{3}(t, s)\right) \bar{\psi}(s) d s \\
\quad-\int_{t_{3}}^{t} \frac{\alpha \eta_{1}(s)}{\rho_{3}^{1 / \alpha}(s)} H_{3}(t, s) \bar{\psi}^{\frac{1+\alpha}{\alpha}}(s) d s \\
\leq H_{3}\left(t, t_{3}\right) \bar{\psi}\left(t_{3}\right)-\int_{t_{3}}^{t} \frac{h_{3}(t, s)}{\rho_{3}(s)} H_{3}^{\frac{\alpha}{\alpha+1}}(t, s) \bar{\psi}(s) d s \\
-\int_{t_{3}}^{t} \frac{\alpha \eta_{1}(s)}{\rho_{3}^{1 / \alpha}(s)} H_{3}(t, s) \bar{\psi}^{\frac{1+\alpha}{\alpha}}(s) d s .
\end{gathered}
$$

By Lemma 1.1, if we set $U=\frac{h_{3}}{\rho_{3}} H_{3}^{\frac{\alpha}{\alpha+1}}, V=\frac{\alpha \eta_{1}}{\rho_{3}^{1 / \alpha}} H_{3}$ and $y=-\bar{\psi}$, then we obtain

$$
-\mathrm{h} \frac{3}{\rho_{3} H_{3}^{\frac{\alpha}{\alpha+1}} \bar{\psi}-\frac{\alpha \eta_{1}}{\rho_{3}^{1 / \alpha}} H_{3} \bar{\psi}^{(\alpha+1) / \alpha} \leq \frac{1}{(\alpha+1)^{\alpha+1}} \frac{h_{3}^{\alpha+1}}{\eta_{1}^{\alpha} \rho_{3}^{\alpha}},}
$$

which with (2.41) gives 
$\frac{1}{H_{3}\left(t, t_{3}\right)} \int_{t_{3}}^{t}\left(k \lambda^{\alpha} H_{3}(t, s) \rho_{3}(s) q(s)-\frac{1}{(\alpha+1)^{\alpha+1}} \frac{h_{3}^{\alpha+1}(t, s)}{\eta_{1}^{\alpha} \rho_{3}^{\alpha}(s)}\right) d s \leq \bar{\psi}\left(t_{3}\right)$, (2.42)

which contradicts $(2.31)$. Assume that Case $\left(\mathbf{C}_{4}\right)$ holds. Proceeding as a proof of Lemma 2.5, we get that $(2.28)$ holds. Next, we define $\bar{\varphi}(t)=$ $\rho_{4}(t) \varphi(t)$ where $\varphi(t)$ defined as $(2.27)$. Then, we note that $\bar{\varphi}(t)<0$ and $\bar{\varphi}^{\prime}(t)=\left(\rho_{4}^{\prime}(t) / \rho_{4}(t)\right) \bar{\varphi}(t)+\rho_{4}(t) \varphi^{\prime}(t)$. Thus, from (2.28), we have

$$
\begin{aligned}
\bar{\varphi}^{\prime}(t) \leq & \frac{\rho_{4}^{\prime}(t)}{\rho_{4}(t)} \bar{\varphi}(t)-\frac{k \widetilde{\mu}^{\alpha}}{2^{\alpha}} \rho_{4}(t) q(t)(1-p(g(t)))^{\alpha} g^{2 \alpha}(t) \\
& -\frac{\alpha}{r^{1 / \alpha}(t) \rho_{4}^{1 / \alpha}(t)} \bar{\varphi}^{\frac{\alpha+1}{\alpha}}(t) .
\end{aligned}
$$

Multiplying this inequality by $H_{4}(t, s)$ and integrating the resulting from $t_{4}$ to $t$, we obtain

$$
\begin{gathered}
\int_{t_{4}}^{t} \frac{k \widetilde{\mu}^{\alpha}}{2^{\alpha}} H_{4}(t, s) \rho_{4}(s) q(s)(1-p(g(s)))^{\alpha} g^{2 \alpha}(s) d s \\
\leq H_{4}\left(t, t_{4}\right) \bar{\varphi}\left(t_{4}\right)+\int_{t_{4}}^{t}\left(\frac{\partial H_{4}(t, s)}{\partial s}+\frac{\rho_{4}^{\prime}(s)}{\rho_{4}(s)} H_{4}(t, s)\right) \bar{\varphi}(s) d s \\
-\int_{t_{4}}^{t} \frac{\alpha}{r^{1 / \alpha}(s) \rho_{4}^{1 / \alpha}(s)} H_{4}(t, s) \bar{\varphi}^{\frac{\alpha+1}{\alpha}}(s) d s
\end{gathered}
$$

and so,

$$
\begin{aligned}
\int_{t_{4}}^{t} \frac{k \widetilde{\mu}^{\alpha}}{2^{\alpha}} H_{4}(t, s) \rho_{4}(s) q(s)(1-p(g(s)))^{\alpha} g^{2 \alpha}(s) \\
\leq H_{4}\left(t, t_{4}\right) \bar{\varphi}\left(t_{4}\right)-\int_{t_{4}}^{t} \frac{h_{4}(t, s)}{\rho_{4}(s)} H_{4}^{\frac{\alpha}{\alpha+1}}(t, s) \bar{\varphi}(s) d s \\
-\int_{t_{4}}^{t} \frac{\alpha}{r^{1 / \alpha}(s) \rho_{4}^{1 / \alpha}(s)} H_{4}(t, s) \bar{\varphi}^{\frac{\alpha+1}{\alpha}}(s) d s .
\end{aligned}
$$

By Lemma 1.1, if we set $U=\frac{h_{4}}{\rho_{4}} H_{4}^{\frac{\alpha}{\alpha+1}}, V=\frac{\alpha}{r^{1 / \alpha} \rho_{4}^{1 / \alpha}} H_{4}$ and $y=-\bar{\varphi}$, then we find

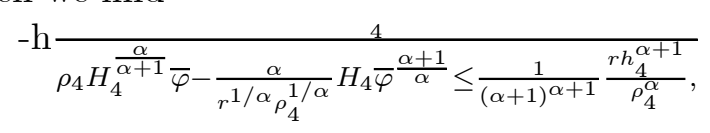


which with (2.45) gives

$$
\frac{1}{H_{4}\left(t, t_{4}\right)} \int_{t_{4}}^{t}\left(\Omega(t, s)-\frac{1}{(\alpha+1)^{\alpha+1}} \frac{r(s)\left[h_{4}^{\alpha+1}(t, s)\right]_{+}}{\rho_{4}^{\alpha}(s)}\right) d s \leq \bar{\varphi}\left(t_{4}\right),
$$

which contradicts (2.31).

Example 2.1. Consider a delay differential equation

$$
\left[e^{t}\left(\left[x(t)+\frac{1}{2} x\left(\frac{t}{2}\right)\right]^{\prime \prime \prime}\right)\right]^{\prime}+\delta e^{t} x\left(\frac{t}{2}\right)=0, \quad t \geq t_{0},
$$

where $\delta>0$ is a constant. We note that

$$
\eta_{i}(t)=e^{-t}, i=0,1,2 .
$$

If we now set $\rho_{1}(t)=\rho_{2}(t)=1$, then we get that $R_{1}=R_{2}=\infty$. Also, we see that $R_{3}=R_{4}>1$ if $\delta>1 / 4$. Thus, By Theorem 2.1, every solution of equation (2.47) is oscillatory for $\delta>1 / 4$.

\section{Acknowledgements}

The authors present their sincere thanks to the editors and anonymous referees. In fact, the observations were very important and contributed significantly to the improvement of the final version of the paper.

\section{References}

[1] M. Bartušek, M. Cecchi, Z. Došlá, and M. Marini, "Fourth-order differenti al equation with deviating argument", Abstract and applied analysis, vol. 2012, Art ID. 185242 , 2012, doi: 10.1155/ 2012/ 185242 
[2] R. P. Agarwal, S. R. Grace, and D. O'Regan, Oscillation theory for difference and functional differential equations. Dordrecht: Springer, 2000, doi: 10.1007/ 978-94-015-9401-1

[3] R. P. Agarwal, C. Zhang, and T. Li, "Some remarks on oscillation of second order neutral differential equations", Applied mathematics and computation, vol. 274, pp. 178-181, 2016, doi: 10.1016/j.amc.2015. 10.089

[4] O. Bazighifan and C. Cesarano, "Some new oscillation criteria for second order neutral differential equations with delayed arguments", Mathematics, vol. 7, no. 7, pp. 619, 2019, doi: 10.3390/math7070619

[5] G. E. Chatzarakis, J. Dzurina, and I. Jadlovska, "New oscillation criteria for second-order half-linear advanced differential equations", Applied mathematics and computation, vol. 347, pp. 404-416, 2019, doi: 10.1016/ j.amc.2018.10.091

[6] G. E. Chatzarakis and I. Jadlovsk, "Improved oscillation results for second-order half-linear delay differential equations", Hacettepe journal of mathematics and statistics, vol. 47, no. 6, 2017, doi: 10.15672/ HJMS.2017.522

[7] C. Cesarano and O. Bazighifan, "Qualitative behavior of solutions of second order differential equations", Symmetry, vol. 11, no. 6, pp. 777, 2019, doi: 10.3390/ sym11060777

[8] S. R. Grace, J. Džurina, I. Jadlovská, and T. Li, “An improved approach for studying oscillation of second-order neutral delay differential equations", Journal of inequalities and applications, vol. 2018, Art ID. 193, doi: 10.1186/ s13660-018-1767-y

[9] S. R. Grace, J. R. Graef, and E. Tunç, "Oscillatory behavior of second order damped neutral differential equations with distributed deviating arguments", Miskolc mathematical notes, vol. 18, no. 2, pp. 759-769, 2017, doi: 10.18514/ MMN.2017.2326

[10] T. Li, Y. V. Rogovchenko, and C. Zhang, "Oscillation results for second order nonlinear neutral differential equations", Advances in difference equations, vol. 2013, Art ID. 336, 2013, doi: 10.1186/1687-18472013-336

[11] O. Moaaz and O. Bazighifan, "Oscillation criteria for second-order quasi-linear neutral functional differential equation", Discrete \& continuous dynamical systems - S, vol. 13, no. 9, pp. 2465-2473, 2020, doi: 10.3934/ dcdss.2020136

[12] O. Moaaz, M. Anis, D. Baleanu, and A. Muhib, "More effective criteria for oscillation of second-order differential equations with neutral arguments", Mathematics, vol. 8, no. 6, Art ID. 986, 2020, doi: 10.3390 / math8060986 
[13] O. Moaaz, G.E. Chatzarakis, T. Abdeljawad, C. Cesarano, and A. Nabih, "Amended oscillation criteria for second-order neutral differential equations with damping term", Advances in difference equations, vol. 2020, Art ID. 553, 2020, doi: 10.1186/ s13662-020-03013-0

[14] R. P. Agarwal, S. R. Grace, and J. V. Manojlovic, "Oscillation criteria for certain fourth order nonlinear functional differential equations", Mathematical and computer modelling, vol. 44, pp. 163-187, 2006, doi: 10.1016/j.mcm.2005.11.015

[15] O. Bazighifan, and C. Cesarano, "A philos-type oscillation criteria for fourth-order neutral differential equations", Symmetry, vol. 12, no. 3, Art ID. 379, 2020, doi: 10.3390/ sym12030379

[16] S. R. Grace, R. P. Agarwal, and J .R. Graef, "Oscillation theorems for fourth order functional differential equations", Journal of applied mathematics and computing, vol. 30, no.2, pp. 75-88, 2009, doi: 10.1007/ s12190-008-0158-9

[17] J. R. Graef, S. R. Grace, and E. Tunç, "Oscillatory behavior of even-order nonlinear differential equations with a sublinear neutral term", Opuscula mathematica, vol. 39, no. 1, pp. 39-47, 2019, doi: 10.7494/ OpMath.2019.39.1.39

[18] T. Li, and Y. V. Rogovchenko, and C. Zhang, "Oscillation of fourth-order quasilinear differential equations", Mathematica bohemica, vol. 140, no. 4, pp.405-418, 2015, doi: 10.21136/ MB.2015.144459

[19] T. Li, and Y. V. Rogovchenko, "Oscillation criteria for even-order neutral differential equations", Applied mathematics letters, vol. 61, pp. 35-41, 2016, doi: 10.1016/j.aml.2016.04.012

[20] O. Bazighifan, F. Minhos, and O. Moaaz, "Sufficient conditions for oscillation of fourth-order neutral differential equations with distributed deviating arguments", Axioms, vol. 9, no. 2, Art ID. 39, 2020, doi: 10.3390/axioms9020039

[21] C. Cesarano, S. Pinelas, F. Al-Showaikh, and O. Bazighifan, "Asymptotic properties of solutions of fourth-order delay differential equations", Symmetry, vol. 11, no. 5, Art ID. 628, 2019, doi: 10.3390/ sym11050628

[22] C. Cesarano, and O. Bazighifan, "Oscillation of fourth-order functional differential equations with distributed delay", Axioms, vol. 8, no. 2, Art ID. 61 , 2019, doi: 10.3390/ axioms8020061

[23] E. M. Elabbasy, C. Cesarano, O. Bazighifan, and O. Moaaz, "Asymptotic and oscillatory behavior of solutions of a class of higher order differential equation", Symmetry, vol. 11, no. 12, Art ID. 1434, 2019, doi: $10.3390 /$ sym11121434 
[24] K. I. Kamo, and H. Usami, "Oscillation theorems for fourth order quasilinear ordinary differential equations", Studia scientiarum mathematicarum hungarica, vol. 39, no. 3-4, pp. 385-406, 2002, doi: 10.1556/ sscmath.39.2002.3-4.10

[25] O. Moaaz, C. Cesarano, and A. Muhib, "Some new oscillation results for fourth-order neutral differential equations", European journal of pure and applied mathematics, vol. 13, no. 2, pp. 185-199, 2020, doi: 10.29020/ nybg.ejpam.v13i2.3654

[26] O. Moaaz, I. Dassios, O. Bazighifan, and A. Muhib, "Oscillation theorems for nonlinear differential equations of fourth-order", Mathematics, vol. 8, no. 4, Art ID. 520, 2020, doi: 10.3390/ math8040520

[27] O. Moaaz and A. Muhib, "New oscillation criteria for nonlinear delay differential equations of fourth-order", Applied mathematics and computation, vol. 377, Art ID. 125192, 2020, doi: 10.1016/j.amc.2020. 125192

[28] O. Moaaz, J. Awrejcewicz, and O. Bazighifan, "A new approach in the study of oscillation criteria of even-order neutral differential equations", Mathematics, vol. 8, no. 2, Art ID. 197, 2020, doi: 10.3390/ math8020197

[29] C. Park, O. Moaaz, and O. Bazighifan, "Oscillation results for higher order differential equations", Axioms, vol. 9, no. 1, Art ID. 14, 2020, doi: 10.3390/ axioms9010014

[30] F. Wu, "Existence of eventually positive solutions of fourth order quasilinear differential equations", Journal of mathematical analysis and applications, vol. 389, no. 1, pp. 632-646, 2012, doi: 10.1016/j.jmaa. 2011.11.061

[31] B. Baculiková and J. Džurina, "On the oscillation of odd order advanced differential equations", Boundary value problems, vol. 2014, Art ID. 214, 2014, doi: 10.1186/ s13661-014-0214-3

[32] B. Baculiková and J. Džurina, "Oscillation of third-order nonlinear differential equations", Applied mathematics letters, vol. 24, no. 4, pp. 466-470, 2011, doi: 10.1016/j.aml.2010.10.043

[33] B. Baculiková and J. Džurina, "Oscillation of third-order functional differential equations", Electronic journal of qualitative theory of differential equations, vol. 2010, Art ID. 43, 2010, doi: 10.14232/ ejqtde. 2010.1.43

[34] O. Moaaz, B. Qaraad, R. A. El-Nabulsi, and O. Bazighifan, "New results for kneser solutions of third-order nonlinear neutral differential equations", Mathematics, vol. 8, no. 5, Art ID. 686, 2020, doi: 10.3390/ math8050686

[35] O. Moaaz, "Oscillatory behavior of solutions of odd-order nonlinear delay differential equations", Advances in difference equations, vol. 2020, Art. ID. 357, 2020, doi: 10.1186/ s13662-020-02821-8 
[36] O. Moaaz, J. Awrejcewicz, and A. Muhib, "Establishing new criteria for oscillation of odd-order nonlineardifferential equations", Mathematics, vol. 8, no. 6, Art ID. 937, 2020, doi: 10.3390/ math8060937

[37] I. T. Kiguradze, and T. A. Chanturia, Asymptotic properties of solutions of nonautonomous ordinary differential equations. Dordrecht: Kluwer, 1993, doi: 10.1007/ 978-94-011-1808-8

[38] C. G. Philos, "Oscillation theorems for linear differential equations of second order", Archiv der mathematik, vol. 53, pp. 482-492, 1989, doi: 10.1007/ BF01324723 